\title{
On the stability of choice processes
}

\section{Eduard Brandstätter*}

Department of Social and Economic Psychology, Johannes Kepler University of Linz, Linz, Austria

${ }^{*}$ Correspondence: eduard.brandstaetter@jku.at
Decisions researchers generally agree that the method of elicitation - be it task format or response mode - has a huge impact on people's responses: different task formats, such as probabilities and frequencies (Gigerenzer and Hoffrage, 1995) - or decisions from description and experience (Hertwig et al., 2004), trigger drastically different behavior. Different response modes, such as choosing, pricing, and matching, have been shown to prompt substantial discrepancies in people's preferences (e.g., Lichtenstein and Slovic, 1971; Tversky et al., 1988). While these streams of research have received much attention, much less is known about the effects of task format in decisions from description, which have been the staple for decision researchers. Here the underlying assumption seems to be that task format has little or no effect on the choice process (Birnbaum, 2004; Birnbaum et al., 2008). When choices differ across experiments, such instabilities can always be modeled by (a) using flexible multi-parameter models that allow for the description of strikingly different choice data (see Brandstätter et al., 2008 for a discussion), or by (b) situating an editing phase prior to the selection phase (Kahneman and Tversky, 1979). In both attempts the core process - the weighting and summing of information - remains unaffected. I argue that both attempts seem unsatisfactory, since different task formats trigger fundamentally different choice processes in decisions from description. Instead of advocating single calculus models I propose an adaptive tool box view of risky choice (Gigerenzer et al., 1999; Brandstätter et al., 2008). The crucial question thus becomes: which task format triggers which choice process? To answer this question I concentrate on decisions from descriptions and on two fundamentally different accounts of risky choice: expected utility theory and its modifications, and the priority heuristic (Brandstätter et al., 2006).

Expected utility theory and its modifications are historically rooted in the work of Daniel Bernoulli, and these models rest on the assumption of weighting and summing of information. Examples are expected utility theory (von Neumann and Morgenstern, 1947), cumulative prospect theory (Tversky and Kahneman, 1992), and the transfer of attention exchange model (Birnbaum et al., 2008). Interpreted as process theories, expected utility theory, for example, predicts that people value payoffs with a utility function, multiply the utilities by the probabilities, sum the products, and finally select the gamble with the higher sum of weighted values. These theories, therefore, predict that people process information within each gamble, such that an overall evaluation for each gamble is made.

The priority heuristic, which represents a different class of models, builds on the work of Luce (1956), Simon (1957), Tversky (1969), and Selten (2001). It is a simple lexicographic semiorder strategy that implies several classic violations of expected utility theory that had previously been accounted for by modifications of expected utility theory (Brandstätter et al., 2006; Katsikopoulos and Gigerenzer, 2008). Across four different data sets with a total of 260 problems, the priority heuristic predicted the majority choice better than each of three modifications of expected utility did. A process test using reaction times further confirmed the heuristic's process predictions (Brandstätter et al., 2006).

To illustrate the heuristic, consider a choice between two simple gambles where each offers "a probability $p$ of winning amount $x$ and a probability $(1-p)$ of winning amount $y$." A choice between two such gambles contains four reasons for choosing: the maximum gain, the minimum gain, and their respective probabilities; because probabilities are complementary, three reasons remain: the minimum gain, the probability of the minimum gain, and the maximum gain. For choices between gambles having two non-negative outcomes (all outcomes are zero or positive), the heuristic consists of the following steps:

Priority rule. Go through reasons in the order of minimum gain, probability of minimum gain, maximum gain.
Stopping rule. Stop examination if the minimum gains differ by $1 / 10$ (or more) of the maximum gain; otherwise, stop examination if probabilities differ by $1 / 10$ (or more) of the probability scale.

Decision rule. Choose the gamble with the more attractive gain (probability).

One-tenth of the maximum gain represents the aspiration level for gains, and 0.1 that for probabilities ${ }^{1}$. Note, the aspiration level for gains is not fixed but changes with the maximum gain of the problem. For probabilities, which are bound between 0 and 1 , the aspiration level of 0.1 is fixed. The term "attractive" refers to the gamble with the higher (minimum or maximum) gain and to the lower probability of the minimum gain. The heuristic does not use any nonlinear transformations of outcomes and probabilities but takes both in their natural currencies (i.e., objective cash amounts and objective probabilities). Unlike the expectation-type models, the priority heuristic predicts that people process information between rather than within gambles. For gambles involving losses, the term "gain" is replaced by "loss." For gambles with more than two outcomes, and gambles involving gains and losses ("mixed gambles"), see Brandstätter et al. (2006).

To illustrate the conceptual difference between an expectation-type model such as prospect theory and the priority heuristic (for details see Brandstätter and Gußmack, submitted), consider the following choice problem (Kahneman and Tversky, 1979).

$$
\begin{array}{ll}
\text { A: } & 6,000 \text { with } p=0.001 \\
& 0 \text { with } p=0.999 \\
\text { B: } & 3,000 \text { with } p=0.002 \\
& 0 \text { with } p=0.998
\end{array}
$$

Most people $(73 \%)$ chose Gamble A. How does prospect theory explain this majority choice? The standard value function is concave for gains, which implies

${ }^{1}$ For the sake of simplicity, I disregard the idea that aspiration levels are rounded (for details see Brandstätter et al., 2006). 
that the larger amount of 6,000 is devalued more than the smaller amount of 3,000 (i.e., compared to a linear value function). The standard value function, thus, predicts B but not A. Prospect theory must explain the choice of A by the overweighting of small probabilities. That is, the overweighting of 0.001 (compared to 0.002) must be stronger than the devaluation of 6,000 (compared to 3,000 ). Because the weighting function is not well-behaved near the endpoints (Kahneman and Tversky, 1979), consider the other possibilities of (a) underweighting, (b) linear weighting, and (c) ignoring small probabilities. Underweighting of small probabilities (captured by an S-shaped rather than an inverse S-shaped probability weighting function) implies the choice of $\mathrm{B}$ but not of $\mathrm{A}$ - as does a linear weighting function (due to the value function). Ignoring small probability outcomes predicts guessing, because the zero outcomes remain. None of these additional possibilities, therefore, can account for the choice of A. The same reasoning holds for cumulative prospect theory, since both prospect theories are identical for two-outcome gambles. According to (cumulative) prospect theory, only the minute difference between 0.001 and 0.002 can cause the choice of A.

For the priority heuristic, this difference is neglected. The heuristic first compares the minimum gains ( 0 and 0 ). Because they do not differ, the probabilities (0.999 and 0.998 or their logical complements 0.001 and 0.002 ) are compared. This difference falls short of the aspiration level (i.e., smaller than 0.1) and people are predicted to choose A, because of its higher maximum gain. Thus, the priority heuristic captures the majority choice by using comparisons between rather than within gambles.

Both the priority heuristic and prospect theory can model the majority choice. In the following I will investigate which of these two models better captures the majority choice. We will see that task format plays a key role in this endeavor. In the light of both models, I also investigate the effect of salience on people's choice processes.

\section{DIFFERENT FORMATS-DIFFERENT PROCESSES \\ FORMAT EFFECTS IN PROCESS TRACING}

Soon after the publication of the priority heuristic, studies investigating information search suggested that the priority heuristic might not capture the choice process (Glöckner and Betsch, 2008; Johnson et al., 2008; Glöckner and Herbold, 2011). Previous tests using eye-tracking or computerized process tracing techniques, such as Mouselab, usually employed neutral information display matrices in which columns represented alternatives, and rows attributes (Ford et al., 1989). Such matrices are neutral, because they favor neither search between nor within alternatives (gambles) or attributes (reasons). The above studies, in contrast, investigated information search by using gambles that were sharply separated: gambles were either placed in extra boxes (Glöckner and Betsch, 2008; Glöckner and Herbold, 2011) or separated by a line (Johnson et al., 2008). Unsurprisingly, such task formats foster search within but not between gambles - thereby favoring expected utility theory and its modifications. Information search measured through eye-tracking or Mouselab, further, may not be equated with information processing measured through think-aloud protocols (Ericsson and Simon, 1993).

To overcome this limitation, we used classic think-aloud protocols to measure the cognitive processes underlying violations of expected utility theory (Brandstätter and Gußmack, submitted). To ensure neutrality in task format, we employed the same format as in Kahneman and Tversky (1979):

$$
\begin{aligned}
& \text { Alternative A } \\
& \begin{array}{cc}
0.1 \% \text { chance to } & \text { Alternative B } \\
\text { win } € 6,000 & 0.2 \% \text { chance to } \\
99.9 \% \text { chance to } & \text { win } € 3,000 \\
\text { win nothing } & 99.8 \% \text { chance to } \\
\text { What would you choose? } & \text { win nothing } \\
\text { A } \bigcirc & \text { B O }
\end{array}
\end{aligned}
$$

It should be noted that this format was intended to support prospect theory - not the priority heuristic. Results show that across all 14 one-stage problems taken from Kahneman and Tversky (1979), most of the protocols revealed that people process information between rather than within gambles - thus lending strong support to processes implied by the priority heuristic (Brandstätter and Gußmack, submitted). That is, it is the difference between 6,000 and 3,000 rather than the overweighting of small probabilities that determines the choice between $€ 6,000$ with probability 0.001 and $€ 3,000$ with 0.002 . Taken together,(a) separated gambles in combination with tools that merely measure information search show that people search information within gambles, whereas (b) neutral task formats in combination with tools that measure deeper cognitive processing suggest that cognitive operations akin to those of the priority heuristic best explain violations of expected utility theory.

\section{SALIENCE EFFECTS}

Salience, I propose, has a strong effect on choice processes. To estimate parameter values for cumulative prospect theory, Tversky and Kahneman (1992) used certainty equivalents inferred from choices. Certainty equivalents represent the amount of money at which a person is indifferent between taking a risky gamble or a sure amount. In their experiments participants made many similar choices between one uncertain prospect and many sure amounts. Hence, the sure amount varied, whereas the probability of the uncertain prospect remained constant (for a similar procedure see, e.g., Tversky and Fox, 1995; Kilka and Weber, 2001; Abdellaou et al., 2005). This method focuses attention on the varying element (i.e., outcomes), while the constant element (i.e., probability) is largely ignored. Why not put things the other way around? What would happen if participants always chose between the same sure amount and a gamble containing varying probability information? Prospect theory's qualitative features might be quite different.

To test this conjecture one study manipulated probability salience (Brandstätter and Kühberger, submitted). To this end, participants estimated the probability of an uncertain event; then they chose between a risky gamble containing the estimated probability (i.e., you win $€ 50$ if the uncertain event occurs, otherwise nothing) and a sure amount of equal expected value. Results differed markedly between the control condition, which contained the same problems in text format, and the probability salience condition. Supporting previous research, in the control condition, participants were riskseeking for low probabilities and risk-averse for high ones. In the probability salience condition, the opposite pattern emerged, and participants were risk-averse for low probabilities and risk-seeking for high ones. 
If winning was unlikely, participants thought that they would not win anyway and selected the sure amount over the gamble; if winning was likely, they chose the risky gamble. These findings suggest a reference point of $p=0.5$ (i.e., winning is likely/unlikely) rather than reference points of $p=0$ and $p=1$. The fact that findings differ substantially for different task formats highlights the error in hoping to uncover (a) a general choice pattern underlying probability weighting and (b) a fixed order of reasons for the priority heuristic.

\section{CONCLUSION}

Decision researchers generally agree that elicitation methods have a strong influence on people's choices. Undoubtedly, in decisions from description, models containing many free parameters or the editing of a choice problem can model task format effects - without changing the core process of weighting and summing. Both attempts seem unsatisfactory. I presented evidence showing that people use the priority heuristic when problems are presented in a neutral text format, but that they search within gambles when gambles are placed in separate boxes. Salience, further, triggers fundamentally different choice processes in decisions from description. Together these results suggest that people use a multitude of decision strategies, and the priority heuristic seems to be one key candidate from the adaptive toolbox of risky-choice-strategies.

\section{REFERENCES}

Abdellaou, M., Vossmann, F., and Weber, M. (2005). Choice-based elicitation and decomposition of deci- sion weights for gains and losses under uncertainty. Manage. Sci. 51, 1384-1399.

Birnbaum, M.H. (2004). Tests of rank-dependent utility and cumulative prospect theory in gambles represented by natural frequencies: Effects of format, event framing, and branch splitting. Organ. Behav. Hum. Decis. Process. 95, 40-65.

Birnbaum, M. H., Johnson, K., and Longbottom, J. L. (2008). Test of cumulative prospect theory with graphical displays of probability. Judgm. Decis. Mak. 3, 528-546.

Brandstätter, E., Gigerenzer, G., and Hertwig, R. (2006). The priority heuristic: making choices without tradeoffs. Psychol. Rev. 113, 409-432.

Brandstätter, E., Gigerenzer, G., and Hertwig, R. (2008). Risky choice with heuristics: reply to Birnbaum (2008), Johnson, Schulte-Mecklenbeck, and Willemsen (2008), and Rieger and Wang (2008). Psychol. Rev. 115, 281-289.

Ericsson, K.A., and Simon, H.A. (1993). Protocol Analysis. Verbal Protocols as Data. Cambridge, MA: MIT Press.

Ford, J. K., Schmitt, N., Schlechtman, S. L., Hults, B. H. and Doherty, M. L. (1989). Process tracing methods: contributions, problems, and neglected research questions. Organ. Behav. Hum. Decis. Process. 43, 75-117.

Gigerenzer, G., and Hoffrage, U. (1995). How to improve Bayesian reasoning without instruction: Frequency formats. Psychol. Rev. 102, 684-704.

Gigerenzer, G., Todd, P. M., and the ABC Research Group. (1999). Simple Heuristics that Make us Smart. New York: Oxford University Press.

Glöckner, A., and Betsch, T. (2008). Do people make decisions under risk based on ignorance? An empirical test of the priority heuristic against cumulative prospect theory. Organ. Behav. Hum. Decis. Process. 107, 75-95.

Glöckner, A., and Herbold, A. K. (2011). An eye-tracking study on information processing in risky decisions: evidence for compensatory strategies based on automatic processes. J. Behav. Decis. Mak. 24, 71-98.

Hertwig, R., Barron, G., Weber, E. U., and Erev, I. (2004). Decisions from experience and the effect of rare events. Psychol. Sci. 15, 534-539.

Johnson, E. J., Schulte-Mecklenbeck, M., and Willemsen, M. C. (2008). Process models deserve process data: a comment on Brandstätter, Gigerenzer, and Hertwig (2006). Psychol. Rev. 115, 263-273.
Kahneman, D., and Tversky, A. (1979). Prospect theory: an analysis of decision under risk. Econometrica 47, 263-291.

Katsikopoulos, K. V., and Gigerenzer, G. (2008). One-reason decision-making. Modeling violations of expected utility theory. J. Risk Uncertain. 37, 35-56.

Kilka, M., and Weber, M. (2001). What determines the shape of the probability weighting function under uncertainty? Manage. Sci. 47, 1712-1726.

Lichtenstein, S., and Slovic, P. (1971). Reversals of preference between bids and choices in gambling decisions. J. Exp. Psychol. 89, 46-55.

Luce, R. D. (1956). Semiorders and a theory of utility discrimination. Econometrica 24, 178-191.

Selten, R. (2001). "What is bounded rationality?" in Bounded rationality: The adaptive toolbox, eds G. Gigerenzer and R. Selten (Cambridge, MA: MIT Press), 13-36.

Simon, H. A. (1957). Models of Man. New York: Wiley.

Tversky, A. (1969). Intransitivity of preferences. Psychol. Rev. 76, 31-48.

Tversky, A., and Fox, C. R. (1995). Weighing risk and uncertainty. Psychol. Rev. 102, 269-283.

Tversky, A., and Kahneman, D. (1992). Advances in prospect theory: Cumulative representation of uncertainty. J. Risk Uncertain. 5, 297-323.

Tversky, A., Sattath, S., and Slovic, P. (1988). Contingent weighting in judgmeint and choice. Psychol. Rev. 95, 1988, 371-384.

von Neumann, J., and Morgenstern, O. (1947). Theory of Gambles and Economic Behavior. Princeton, NJ: Princeton University Press.

Received:07 July 2011; accepted: 11 October 2011; published online: 15 November 2011.

Citation: Brandstätter E (2011) On the stability of choice processes. Front. Psychology 2:295. doi: 10.3389/ fpsyg.2011.00295

This article was submitted to Frontiers in Cognition, a specialty of Frontiers in Psychology.

Copyright $(\odot 2011$ Brandstätter. This is an open-access article subject to a non-exclusive license between the authors and Frontiers Media SA, which permits use, distribution and reproduction in other forums, provided the original authors and source are credited and other Frontiers conditions are complied with. 